\title{
Steerable Intravitreal Inserts for Drug Delivery: In Vitro and Ex Vivo Mobility Experiments*
}

\author{
Christos Bergeles $^{1}$, Michael P. Kummer ${ }^{1}$, Bradley E. Kratochvil ${ }^{1}$, \\ Carsten Framme ${ }^{2}$, and Bradley J. Nelson ${ }^{1}$ \\ 1 Institute of Robotics and Intelligent Systems, \\ ETH Zurich, 8092 Zurich, Switzerland \\ cbergeles@ethz.ch \\ 2 Universitätspital Bern, Inselspital, 3010 Bern, Switzerland
}

\begin{abstract}
The progress of wet age-related macular degeneration can now be controlled by intravitreal drug injection. This approach requires repeated injections, which could be avoided by delivering the drug to the retina. Intraocular implants are a promising solution for drug delivery near the retina. Currently, their accurate placement is challenging, and they can only be removed after a vitrectomy. In this paper, we introduce an approach for minimally invasive retinal drug delivery using magnetic intraocular inserts. We briefly discuss the electromagnetic-control system for magnetic implants and then focus on evaluating their ability to move in the vitreous humor. The mobility of magnetic intraocular implants is estimated in vitro with synthesized vitreous humors, and ex vivo with experiments on cadaver porcine eyes. Preliminary results show that with such magnetic implants a vitrectomy can be avoided.
\end{abstract}

\section{Age-Related Macular Degeneration}

Age-related macular degeneration (AMD) is a degenerative disorder of the posterior eye segment that is characterized by the development of lesions in the macular area. Figure 1 illustrates how the disease progresses. The result is loss in central-vision acuity.

AMD is the leading cause of blindness worldwide. In the United States, for example, it accounts for more than $50 \%$ of the cases of blindness, and, given the aging of the population, the number of affected people is expected to double [1]. Improving the efficacy of treatment methods for AMD can improve the quality of life for a significant number of patients.

\section{Existing Therapies and Challenges}

Current treatments for AMD aim at delivering drugs to inhibit the formation of new lesions and prevent further vision loss [2]. The most targeted drug delivery

\footnotetext{
* This work was supported by the NCCR Co-Me of the Swiss National Science Foundation.
} 


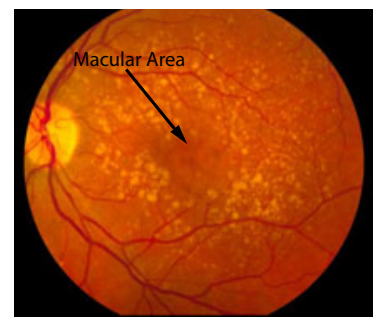

(a)

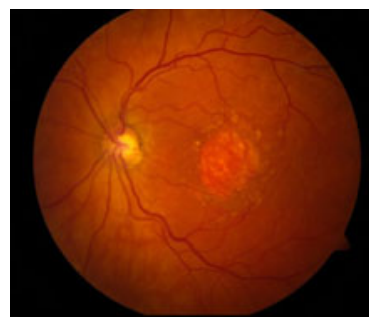

(b)

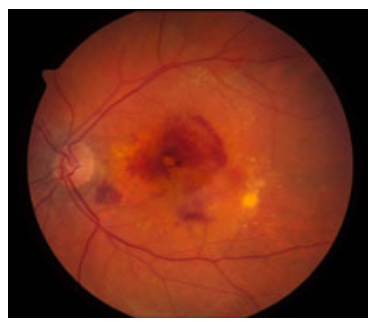

(c)

Fig. 1. Retinal-fundus images showing different stages of AMD: (a) AMD at an intermediate stage, (b) geographic atrophy associated with AMD, and (c) angiogenesis in AMD. (Images from the National Eye Institute, National Institutes of Health, USA).

method is intravitreal administration [3] and has been recently introduced for AMD stabilization. However, repeated injections are required for effective treatment, since the physiology of the vitreous humor reduces the amount of drug that finally reaches the macular area.

Biodegradable and non-biodegradable inserts that act as drug reservoirs are a proposed solution to this problem [4. The reservoirs can be tailored to allow prolonged drug release, and placement of the inserts close to the retina enables targeted drug delivery. Non-biodegradable drug reservoirs are preferable due to their more controlled release mechanisms [5].

Non-biodegradable inserts must be surgically removed after their reservoir is depleted. This requires a vitrectomy, a risky invasive procedure that involves dissecting and replacing the vitreous humor with a thin solution. This requirement hinders the acceptance of the intraocular-implant treatment by patients.

\section{Electromagnetically Steerable Intravitreal Inserts}

Microrobotic devices are an emerging tool for minimally invasive surgery and localized drug delivery [6. In this paper, the use of electromagnetically controlled microrobots that act as intravitreal inserts with drug reservoirs is proposed (Fig. 2(a). Mobility allows these devices to be positioned and removed in a controllable and minimally invasive manner, potentially avoiding a vitrectomy. The microrobots are introduced in the posterior eye segment through the pars plana region of the sclera and are wirelessly controlled to the macula where they release their payload. Upon depletion of their reservoir they are controlled towards the entry point for removal by a magnetic tool. For AMD treatment, the microrobots can be placed in the area of the lower vessel arcade without severely affecting sight.

The microrobots have an outer diameter of less than $500 \mu \mathrm{m}$ and fit in a $23 \mathrm{G}$ needle. Their length, i.e. their reservoir, can be tailored based on the dosing required. Initially, the microrobots were microassembled soft-magnetic elliptical pieces [7, but microrobots from permanent magnetic materials are an alternative 


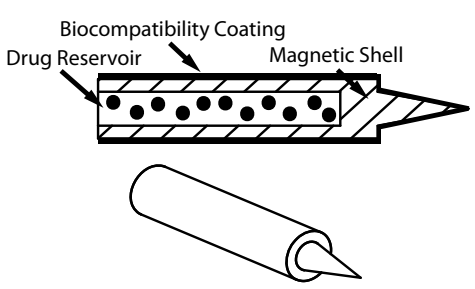

(a)

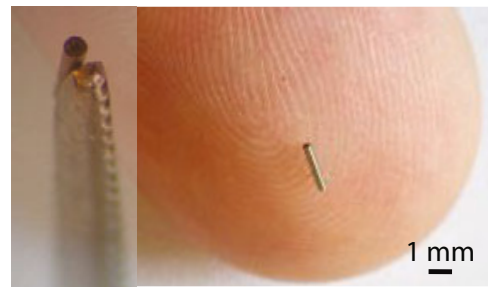

(b)

Fig. 2. (a) Concept of a magnetically driven reservoir, and (b) a $2.4 \mathrm{~mm}$ long and $0.38 \mathrm{~mm}$ wide hollow SmCo microrobot ( $0.135 \mathrm{~mm}$ inner diameter), similar to pSivida's Illuvien $\mathbb{R}$

that is under examination (Fig. 2(b) ). Researchers are also investigating the intissue mobility of screw-like devices for drug delivery 8. Scaling these devices to the sub-mm range present fabrication challenges. Our initial experiments indicate that rotating screw-like devices get tangled up in the collagen fibers of the vitreous humor, thus, screw motion is not considered in this work.

These microrobotic devices are non-bioerrodable, and are rendered biocompatible using polypyrrole coatings [9]. Since the human eye, in general, has "immune privileges" that limit the risk of an inflammatory reaction to foreign antigens [10], severe reactions from the immune system due to the presence of coated microdevices is not expected.

\section{Five Degree-of-Freedom Electromagnetic Steering}

Unrestrained wireless electromagnetic control was introduced in 11, where the researchers described the OctoMag (Fig. 3), an electromagnetic control system capable of controlling magnetic devices in five degrees-of-freedom (DOF) (3-DOF position, 2-DOF pointing orientation). The workspace of the OctoMag is approximately $15 \mathrm{~mm} \times 15 \mathrm{~mm}$ and covers the posterior segment of the human eye. The system consists of eight DC-operated electromagnets arranged in a hemispherical configuration. The electromagnets are equipped with soft-magnetic cores, and, assuming that the cores are from a near-ideal material, the fields of all the magnets superimpose linearly. The introduction of soft-magnetic cores enables the generation of high field-gradients. In its current configuration, a small animal (e.g. a rabbit) can be placed between the electromagnets for in vivo experiments. Manual position control of the microdevices is preferred by surgeons and is enabled by the OctoMag's interface.

Currently, the OctoMag can create a maximum gradient of $1.5 \frac{\mathrm{T}}{\mathrm{m}}$. Microdevices experience different forces when introduced to the same electromagnetic field gradient due to their different magnetic properties. Experimental estimation of the maximum applicable force indicates how they can interact with the intraocular environment. 


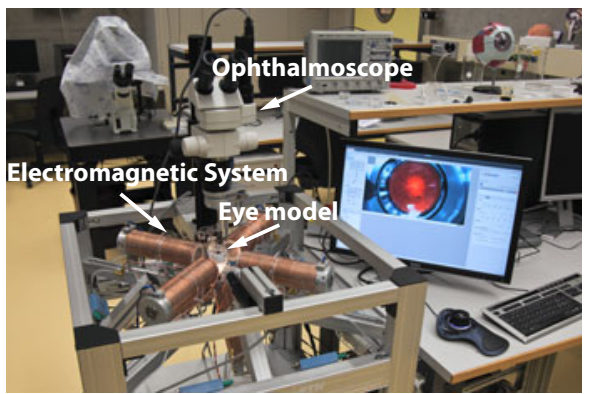

(a)

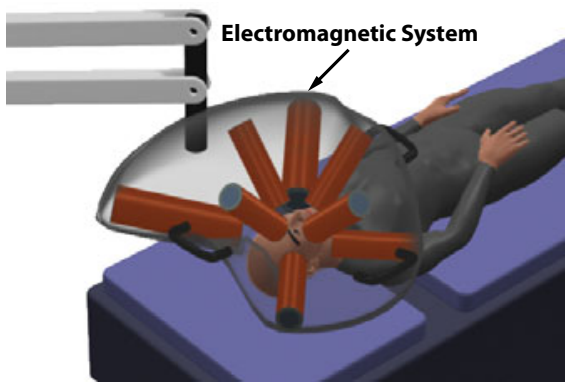

(b)

Fig. 3. (a) The OctoMag electromagnetic control system with an eye model in its workspace, and (b) rendering of a future system for AMD treatment

Table 1. Maximum force on $\mathrm{NdFeB}$, and respectively, SmCo devices for various orientations

\begin{tabular}{|c||c|c|c|c|c|}
\hline $\begin{array}{c}\text { Field } \\
\text { Orientation }\end{array}$ & $\begin{array}{c}\mathbf{F}_{\text {up }} \\
(\mu \mathrm{N})\end{array}$ & $\begin{array}{c}\mathbf{F}_{\text {down }} \\
(\mu \mathrm{N})\end{array}$ & $\begin{array}{c}\mathbf{F}_{\text {lat, } \mathrm{x}} \\
(\mu \mathrm{N})\end{array}$ & $\begin{array}{c}\mathbf{F}_{\text {lat,y }} \\
(\mu \mathrm{N})\end{array}$ & $\begin{array}{c}\mathbf{F}_{\text {lat,xy }} \\
(\mu \mathrm{N})\end{array}$ \\
\hline \hline$z$ & $287.0,365.0$ & $287.0,365.0$ & $233.9,209.1$ & $233.9,209.1$ & $287.0,296.3$ \\
\hline$z$ & $287.0,365.0$ & $287.0,365.0$ & $233.9,209.1$ & $233.9,209.1$ & $287.0,296.3$ \\
\hline$x$ & $287.0,261.4$ & $287.0,261.4$ & $287.9,387.3$ & $287.0,387.3$ & $287.0,331.1$ \\
\hline$x y$ & $287.0,361.2$ & $287.0,361.2$ & $287.0,369.9$ & $287.0,369.9$ & $287.0,364.1$ \\
\hline
\end{tabular}

For a magnetic field of $\|\mathbf{B}\|=15 \mathrm{mT}$, the maximum generated forces on $\mathrm{NdFeB}$ and SmCo inserts were estimated (Table 1). The maximum force applied to soft-magnetic $\mathrm{Ni}$ or CoNi microrobots along every orientation is $125.7 \mu \mathrm{N}$ [1]. The superior performance of permanent magnetic microrobots makes them more attractive for use as intravitreal drug carriers. According to [12, 75\% of the forces in retinal surgery are between 0 and $7.5 \mathrm{mN}$. Being on the extreme lower range of this scale, no risk of accidental retinal penetration or tears from using permanent magnetic microrobots is expected.

\section{Evaluating the Mobility of Steerable Inserts In Vitro}

In [13, a protocol to create artificial vitreous humors with desired viscosity (loss modulus, G") and elasticity (storage modulus, G') was introduced. These artificial humors consist of water $\left(\mathrm{H}_{2} \mathrm{O}\right)$, agar-agar $(\mathrm{AG})$, and hyaluronic acid (HA). Water is $99 \%$ of the natural vitreous humor, and hyaluronic acid gives it some of its mechanical properties [14]. Agar is giving elasticity to the mixture.

Eleven fluids with different properties were synthesized (Table 2). These artificial vitreous humors cover a range from a mechanical equivalent of the porcine vitreous humor to human vitreous humor cases reported in [14. The storage modulus for the vitreous of these human eyes is shown in Table 3. Mechanical 


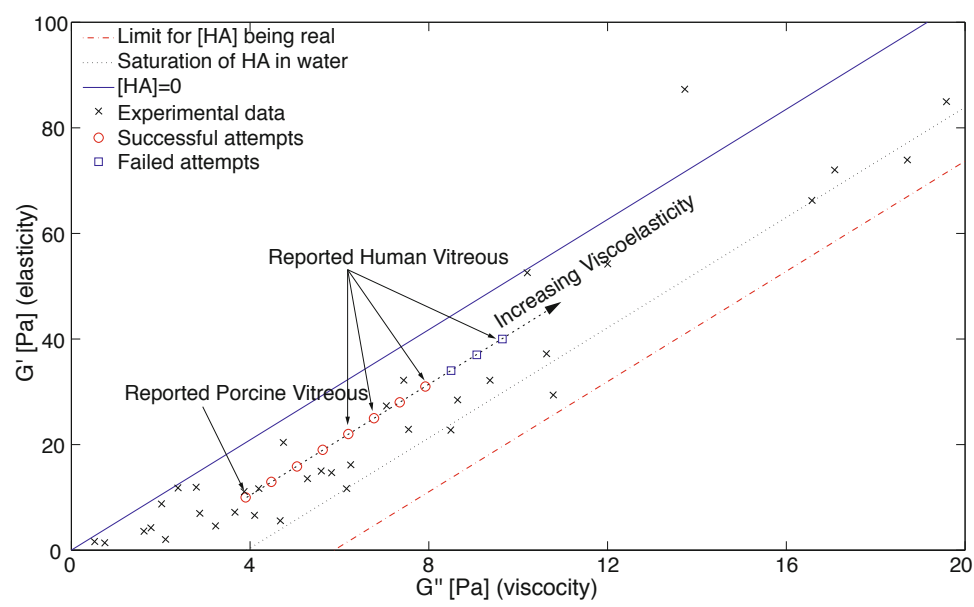

Fig. 4. Motion experiments with artificial vitreous humor. The storage and loss moduli are from 13 . The red circles indicate successful movement attempts and the blue boxes indicate that the insert was unable to translate. The crosses are the different fluids examined in 13 to extract the relationship between AG, HA, storage and loss. Image adapted from [13.

Table 2. Agar for $10 \mathrm{~mL}$ of water. HA amount is fixed at $2.21 \mathrm{mg} / \mathrm{mL}$.

\begin{tabular}{|c||c|c|c|c|c|c|c|c|c|c|c|}
\hline Fluid Case & $\mathbf{1}$ & $\mathbf{2}$ & $\mathbf{3}$ & $\mathbf{4}$ & $\mathbf{5}$ & $\mathbf{6}$ & $\mathbf{7}$ & $\mathbf{8}$ & $\mathbf{9}$ & $\mathbf{1 0}$ & $\mathbf{1 1}$ \\
\hline \hline AG $(\mathbf{m g} / \mathbf{m L})$ & 1.098 & 1.27 & 1.42 & 1.56 & 1.68 & 1.80 & 1.91 & 2.014 & 2.11 & 2.21 & 2.30 \\
\hline
\end{tabular}

data of the human-eye vitreous are scarce in the literature. It is known, however, that the vitreous liquifies with age [15, and this trend can also be observed in Table 3. These data enables us to synthesize fluids for experiments that provide an indication of how microdevices will behave in human vitreous humor.

According to 13 , we can fix the amount of HA in solution and vary only the amount of AG to achieve desired mechanical properties. The substances are added in water heated to boiling. The solution is maintained near boiling, stirred rigorously until fully transparent, and left to cool overnight. Figure 4 shows the storage and loss moduli for different synthesized vitreous humors. Circles and squares indicate successful and failed attempts, respectively.

Experiments were conducted with $2 \mathrm{~mm}$-long CoNi microrobots, and $1 \mathrm{~mm}$ long NdFeB microrobots. The devices were servoed along their $x$-axis using the OctoMag as this orientation results in a lower fluidic resistance. We observed that even in the thinnest of the synthesized fluids, a fluid with similar viscoelastic properties to the porcine vitreous, the CoNi microrobot is unable to move. The $\mathrm{NdFeB}$ microrobot is able to move in a greater range of mediums, ranging from the porcine vitreous to reported human values. These human values correspond to ages 45 and 62 in Table 3 , and are within the age group that the developed drug delivery method is intended for. 
Table 3. Storage modulus and age for different human eyes. "L" and "R" correspond to the left and right eye respectively. Data extracted from 14].

\begin{tabular}{|c||c|c|c|c|c|c|c|c|}
\hline \multicolumn{1}{|c||}{} & \multicolumn{2}{|c|}{ Patient 1 } & \multicolumn{2}{|c|}{ Patient 2 } & \multicolumn{2}{|c|}{ Patient 3 } & Patient 4 \\
\hline Age & \multicolumn{2}{|c|}{20} & \multicolumn{2}{|c|}{30} & \multicolumn{2}{|c|}{45} & \multicolumn{2}{|c|}{62} \\
\hline Eye & L & R & L & R & L & R & L & R \\
\hline \hline G' $[\mathbf{P a}]$ & 30 & 27 & 39 & 5 & 27 & 25 & 15 & 3 \\
\hline
\end{tabular}

To summarize, it is difficult to use CoNi microdevices intraocularly without a vitrectomy, but based on the experimental results, this is possible with $\mathrm{Nd}$ FeB microrobots. This is not surprising since higher forces can be exercised on permanent-magnetic microrobots than on soft-magnetic ones.

\section{Evaluating the Mobility of Steerable Inserts Ex Vivo}

In Table 3, it can be seen that the vitreous humor of the 62 year old donor has a storage modulus similar to that of the porcine vitreous humor. Additionally, a study performed in [16] reports that the central vitreous humor of the human and the pig are rheologically similar. The same study states that the anterior and posterior regions of the porcine vitreous resemble a "thick" gel, whereas the human vitreous has a "thinner" and in some cases "watery" consistency. We can conclude that, with respect to the properties of the vitreous humor, porcine eyes can be used as a "hard" model of the human eye.

Studies on the human vitreous humor are conducted with eyes from elderly donors, whereas porcine eyes are taken from relatively young animals. This fact is also acknowledged in [16. From personal communication with vitreoretinal surgeons, it was understood that successful mobility of microrobots in porcine eyes received from slaughterhouses will be a good indicator for similar or better mobility performance in the eyes of elderly human patients.

Thus, the goal is to evaluate the mobility of the microdevices in porcine vitreous humor. In [14], it is stressed that the vitreous humor rapidly loses hyaluronic acid upon removal from the eye globe, it collapses, and its mechanical properties change significantly. Thus, mobility experiments need to be performed in the eye globe. As in [12, the cadaver eye is dissected above the pars plana region, and the anterior chamber and iris are exposed after removing the cornea. Subsequently, the intraocular lens and iris are removed, and the vitreous humor is accessible. Removing the optical elements facilitates visualization, and no ophthalmoscope is required for observation.

The microrobot is inserted in the vitreous humor using a syringe equipped with a glass pipette. Afterwards, the eye is positioned under the OctoMag for experimentation. The eye is illuminated transsclerally, and the images are captured by a Leica M80 microscope equipped with a Grasshopper-14S5M/C camera.

Results for a NdFeB microrobot moving in the vitreous humor of two cadaver porcine eyes are shown in Fig. 5. The mobility of the microrobots supports the 

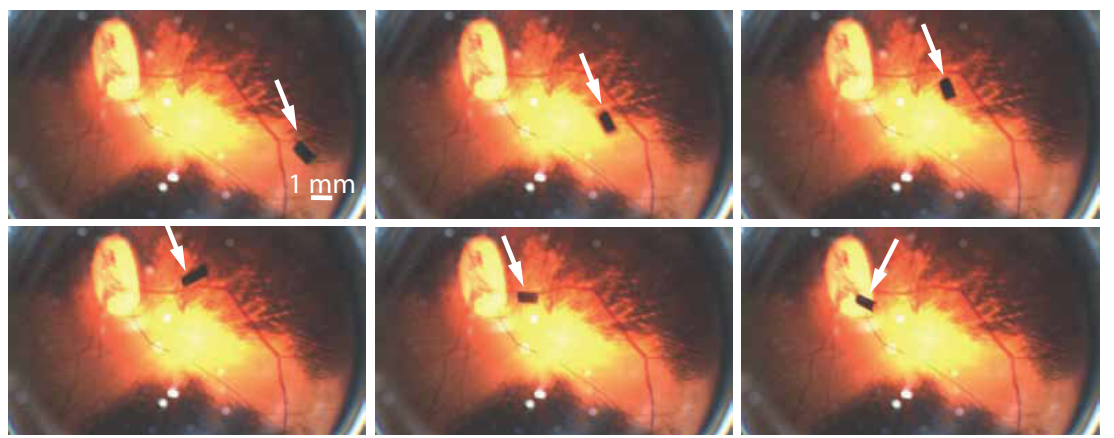

(a)
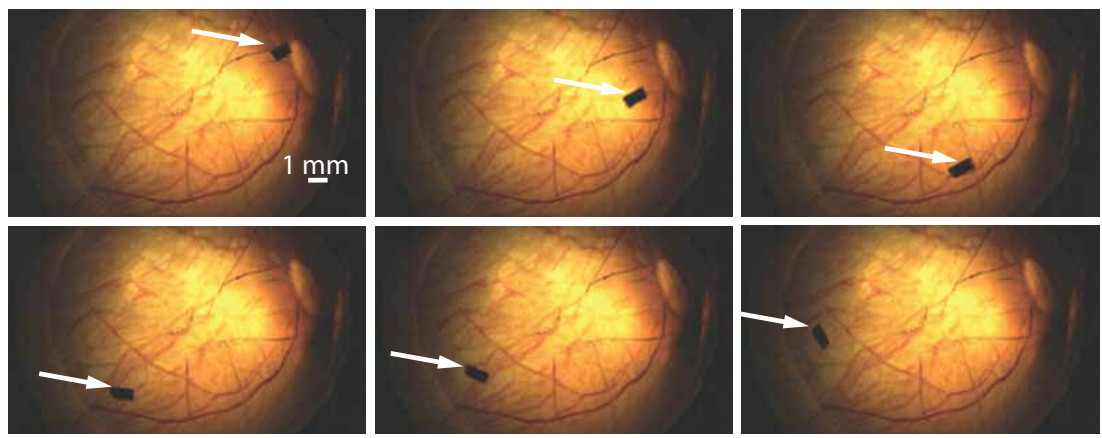

(b)

Fig. 5. Sequences of frames showing a $1 \mathrm{~mm}$ long NdFeB microrobot moving in the vitreous humor of two cadaver porcine eyes

in vitro experimental results and suggests that magnetic microrobotic inserts can be used in human eyes without necessitating a vitrectomy. This, together with the increased mobility the electromagnetic control offers, will enable the minimally invasive delivery of existing drugs closer to the retina.

\section{Conclusions and Future Work}

In this paper, the concept of performing minimally invasive localized drug delivery in the posterior eye segment using steerable microrobotic drug carriers was introduced. The aim of the current technology is to assist in the treatment of age-related macular degeneration, however, it can also be used for other posterior-eye diseases. Magnetic microrobots can be considered a safe tool for localized drug delivery. In vitro experimentation demonstrated that permanentmagnetic microrobots are able to move in a range of fluids covering porcine vitreous and human vitreous. Successful ex vivo experiments lead to the conclusion that magnetic microrobots have the potential to be wirelessly controlled in human eyes without a vitrectomy, making drug delivery in the posterior-eye segment more targeted and less invasive. Future work will include a statistical analysis of repeated experiments and design optimization of the microdevices. 


\section{References}

1. Congdon, N., O'Colmain, B., Klaver, C.C., Klein, R., Munoz, B., Friedman, D.S., Kempen, J., Taylor, H.R., Mitchell, P.: Causes and prevalence of visual impairment among adults in the United States. Arch. Ophthalmology 122(4), 477-485 (2004)

2. Nowak, J.Z.: Age-related macular degeneration: pathogenesis and therapy. Pharmacological Reports 58(3), 353-363 (2006)

3. Lee, S.S., Robinson, M.R.: Novel drug delivery systems for retinal diseases. Ophthalmic Research 41(3), 124-135 (2009)

4. Danckwerts, M.P., Fassihi, A.: Implantable controlled release drug delivery systems. Drug Development and Industrial Pharmacy 17(11), 1465-1502 (1991)

5. Yasukawa, T., Ogura, Y., Tabata, Y., Kimura, H., Wiedemann, P., Honda, Y.: Drug delivery systems for vitreoretinal diseases. Progress in Retinal and Eye Research 23(3), 253-281 (2004)

6. Nelson, B.J., Kaliakatsos, I.K., Abbott, J.J.: Microrobots for minimally invasive medicine. Annual Review of Biomedical Engineering 12, 55-85 (2010)

7. Yeşin, K.B., Vollmers, K., Nelson, B.J.: Modeling and control of untethered biomicrorobots in a fluidic environment using electromagnetic fields. Int. J. Robotics Research 25(5-6), 527-536 (2006)

8. Ishiyama, K., Sendoh, M., Yamazaki, A., Arai, K.I.: Swimming micro-machine driven by magnetic torque. Sensor and Actuators A: Physical 91(1-2), 141-144 (2001)

9. Sivaraman, K.M., Bayrakceken, K., Ergeneman, O., Pane, S., Lühmann, T., Hall, H., Nelson, B.J.: Tailoring the drug loading capacity of polypyrrole films for use in intraocular biomicrorobots. In: IEEE Int. Conf. Engineering in Medicine and Biology, pp. 4359-4362 (2010)

10. Choonara, Y.E., Pillay, V., Danckwerts, M.P., Carmichael, T.R., Du Toit, L.C.: A review of implantable intravitreal drug delivery technologies for the treatment of posterior segment eye diseases. J. Pharmaceutical Sciences 99(5), 2219-2239 (2010)

11. Kummer, M.P., Abbott, J.J., Kratochvil, B.E., Borer, R., Sengul, A., Nelson, B.J.: OctoMag: An electromagnetic system for 5-DOF wireless micromanipulation. IEEE Trans. Robotics 26(6), 1006-1017 (2010)

12. Gupta, P.K., Jensen, P.S., de Juan Jr., E.: Surgical forces and tactile perception during retinal microsurgery. In: Taylor, C., Colchester, A. (eds.) MICCAI 1999. LNCS, vol. 1679, pp. 1218-1225. Springer, Heidelberg (1999)

13. Kummer, M.P., Abbott, J.J., Dinser, S., Nelson, B.J.: Artificial vitreous humor for in vitro experiments. In: IEEE Int. Conf. Engineering in Medicine and Biology, pp. 6406-6409 (2007)

14. Nickerson, C.S.: Engineering the mechanical properties of ocular tissues. PhD thesis, California Institute of Technology (2005)

15. Los, L.I., Van der Worp, R.J., Van Luyn, M.J.A., Hooymans, J.M.M.: Age-related liquefaction of the human vitreous body: LM and TEM evaluation of the role of proteoglycans and collagen. Investigative Ophthalmology and Visual Science 44(7), 2828-2833 (2003)

16. Lee, B., Litt, M., Buchsbaum, G.: Rheology of the vitreous body: Part 2. viscoelasticity of bovine and porcine vitreous. Biorheology 31(4), 327-338 (1994) 